\title{
Photoluminescence of Hexagonal Boron Nitride (h-BN) Film
}

\author{
Moon-Seog Jin* and Nam-Oh Kim ${ }^{\dagger}$

\begin{abstract}
Hexagonal boron nitride (BN) films were prepared. The process involved, spraying $\mathrm{BN}$ powder-dispersed $\mathrm{H}_{3} \mathrm{BO}_{4}-\mathrm{BCl}_{3}$-ethyl alcohol solution on quartz plates, and the drying off quartz plates before, and annealing at $1070{ }^{\circ} \mathrm{C}$ in a nitrogen atmosphere. The optical energy band gap of the BN films was $5.28 \mathrm{eV}$. Photoluminescence peaks with energies of 3.44, 3.16, 2.97, and $2.35 \mathrm{eV}$ at $10 \mathrm{~K}$ were observed and analyzed. Accordingly, these have resulted from donor-acceptor pair recombinations.
\end{abstract}

Keywords: BN (boron-nitride), Energy gap, Photoluminescence, Recombinations

\section{Introduction}

Boron nitride (BN) semiconductors with ultra-wideenergy band gaps are of two types: namely, cubic BN (c$\mathrm{BN}$ ) and hexagonal $\mathrm{BN}$ (h-BN), which are structurally similar to diamond and graphite, respectively [1]-[3]. The h-BN film type has received much attention because of similarities to carbon and its promising properties such as good chemical and mechanical stability. Optical properties [1]-[4] and deep levels [5], [6] of h-BN prepared by various methods have been explored because the relatively easier in preparation of h-BN compared with c-BN. In contrast, few studies on the photoluminescence (PL) spectra of h-BN have been reported. Meanwhile, the PL transition mechanism of h-BN has not yet been reported to date. Based on the discussion above, we coated a BN film into the inner part of a quartz tube. Thereafter, the quartz tube was used to grow single-crystal semiconductors without impurities. Next, we coated BN film into quartz plates. This process allowed us to obtain well-grown h-BN films. Then, we explored the PL mechanism of h-BN films by investigating the crystal structures and optical energy band gaps. Finally, PL spectra measurement was conducted. The PL transition mechanism of h-BN was also identified.

\section{Experimental}

We dispersed $\mathrm{BN}$ powder (purity 99.99\%, nanocrystalline) with a hexagonal structure into a $\mathrm{H}_{3} \mathrm{BO}_{4}-\mathrm{BCl}_{3}$ ethyl alcohol solution. The solution was sprayed on quartz plates and then dried off. This process was repeated several times with sprayed thickness of about $1 \mu \mathrm{m}$. The sprayed area was $1.5 \times 1.5 \mathrm{~cm}^{2}$. The dried quartz plates were placed in a tubular electric furnace, heated slowly to $1070{ }^{\circ} \mathrm{C}$ with

\footnotetext{
$\dagger \quad$ Corresponding Author: Dept. of Electricity at Chosun University College of Science \& Technology, Korea. (nokim88@nate.com)

* Dept. of Optometry and Optic Science, Dongshin Univerity, Korea. (msjin@dsu.ac.kr)

Received: March 23, 2010; Accepted: July 21, 2010
}

nitrogen gas blowing, and annealed for $96 \mathrm{~min}$. The grown $\mathrm{BN}$ films on the quartz plates were translucent.

The crystal structure of the grown BN films was investigated by analyzing their X-ray diffraction patterns. Optical absorption spectra were measured by using a uv-vis-nir spectrophotometer (Hitachi, U-3501) equipped with 60$\mathrm{mm}$ integrating sphere. Optical absorption spectra were measured with nitrogen gas blowing into a sample chamber at the wavelength range of $187-385 \mathrm{~nm}$. Photoluminescence spectra were measured by a conventional PL measurement system consisting of a double monochromator (Spex1403, $\mathrm{f}=0.85 \mathrm{~m}$ ), a photomultiplier tube (RCA C31034), and a cryogenic system (APD, SH-4). The 1064nm line of the Nd-YAG laser (Spectra Physics, T10-85) and the 266-nm line by redoubling the 1064-nm line were used as sources of excitation.

\section{Results and Discussion}

\subsection{Crystal Structure and Defect Levels of the Hex- agonal BN Film}

Fig. 1 shows the X-ray diffraction pattern of the grown $\mathrm{BN}$ film. The BN film crystallized into an h-BN structure from the intensive diffraction peaks of (002), (100), (102), (110), and (112). Based on analysis of the diffraction pattern, the lattice constants of the h-BN film were $\mathrm{a}=2.5044$ $\pm 0.0025 \AA$ and $\mathrm{c}=6.6562 \pm 0.0054 \AA$.

Thermally stimulated current (TSC) method [7] was used obtain the defect levels in the h-BN film. Three donor levels and three acceptor levels were obtained. The activation energies of the donor levels were $0.63,1.10$, and 1.51 $\mathrm{eV}$, while the activation energies of the acceptor levels were $1.02,1.21$, and $1.42 \mathrm{eV}$. A comparison of these results with those by Lopatin and Konusov [6], is indicative of the reasonable values for the donor and acceptor levels.

The SEM surfaces micrograph in Fig. 2 shows the formation of the h-BN film in the layer structure. The film surface is rough with grainy layer morphology. The grain size of the film was with an average grain size of about $3 \mu \mathrm{m}$. 


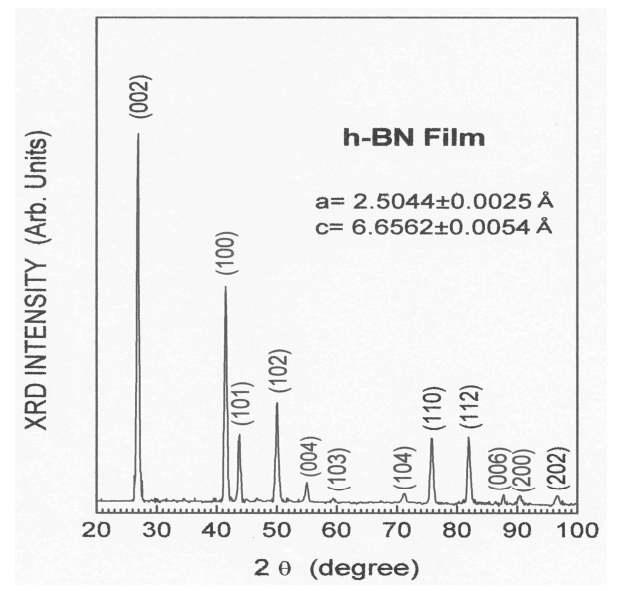

Fig. 1. Powdered X-ray diffraction pattern of the h-BN film.

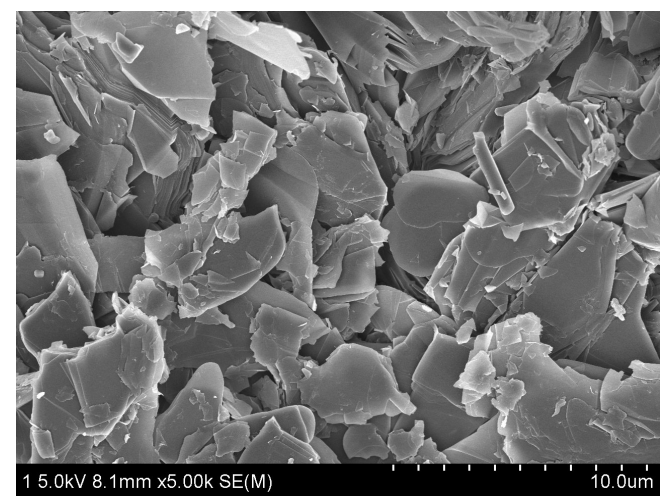

Fig. 2. SEM surface photographs of the h-BN film.

\subsection{Photoluminescence Characteristics of Hexagonal BN Film}

Fig. 3 shows the optical absorption spectrum of the h$\mathrm{BN}$ film at $293 \mathrm{~K}$, presenting a peak at the ultraviolet wavelength range and an optical absorption edge at 234.8 $\mathrm{nm}(5.28 \mathrm{eV})$. The optical absorption edge corresponds to the optical energy band gap of the h-BN film. The optical energy band gap of $5.28 \mathrm{eV}$ agrees well with $5.2 \mathrm{eV}$ reported by Hoffman et al. [2] and $5.14 \mathrm{eV}$ by Stenzel et al. [3]. In Fig. 2 shows an absorption peak located at $263 \mathrm{~nm}$ $(4.71 \mathrm{eV})$, which is believed to be a result of a defect level in the h-BN film. The peak energy of this absorption peak corresponds to the difference between the optical energy band gap $(5.28 \mathrm{eV})$ and the activation energy of the donor level $(0.63 \mathrm{eV})$ as mentioned in the previous paragraph. Accordingly, the absorption peak at $263 \mathrm{~nm}$ was influenced by the donor level $0.63 \mathrm{eV}$, as observed by the TSC method.

Fig. 4 shows the PL spectra of the h-BN film at 300 and $10 \mathrm{~K}$. The PL spectrum at $300 \mathrm{~K}$ presented two broad PL peaks at the wavelength ranges of $400-430 \mathrm{~nm}$ and $500-$ $550 \mathrm{~nm}$. To investigate the PL peaks in detail, we measured the PL spectrum at $10 \mathrm{~K}$. The PL spectrum at $10 \mathrm{~K}$ exhibited three peaks at the wavelength range of $345-450 \mathrm{~nm}$ and another peak at the range of $460-600 \mathrm{~nm}$, their corresponding values $360.4 \mathrm{~nm}(3.44 \mathrm{eV}, \mathrm{P} 1), 392.4 \mathrm{~nm}(3.16 \mathrm{eV}$,

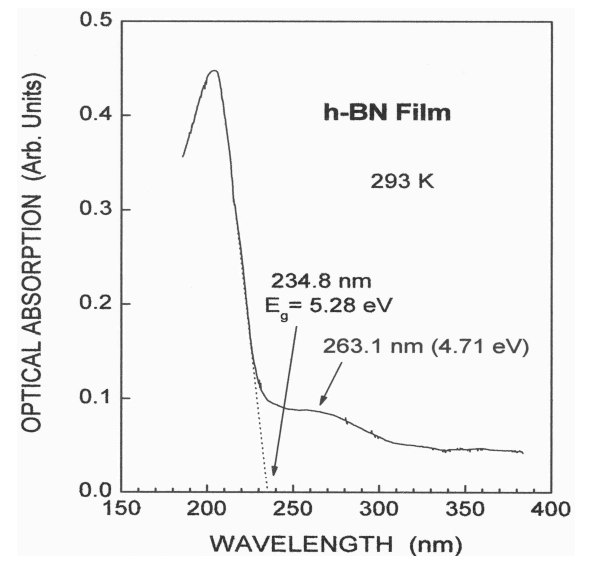

Fig. 3. Optical absorption spectrum of the h-BN film at $293 \mathrm{~K}$.

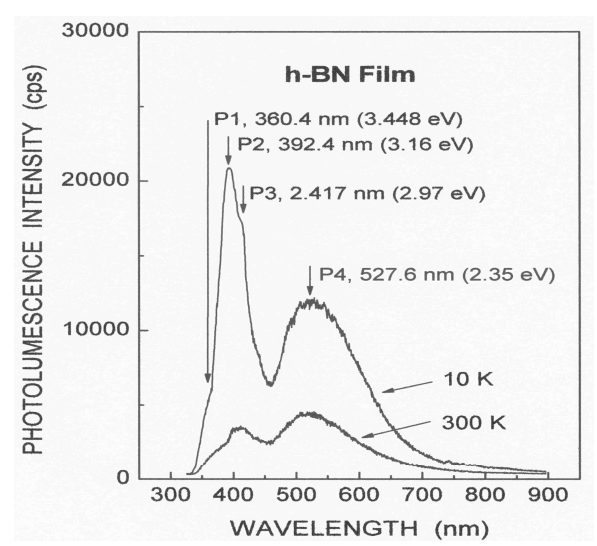

Fig. 4. Photoluminescence spectra of the h-BN film at 10 and $300 \mathrm{~K}$.

$\mathrm{P} 2)$, and $417.5 \mathrm{~nm}(2.97 \mathrm{eV}, \mathrm{P} 3)$, and the other peak at $527.6 \mathrm{~nm}(2.35 \mathrm{eV}, \mathrm{P} 4)$.

In accordance with the donor and acceptor levels and the optical energy band gap of the h-BN film, the PL peaks (P1, P2, P3, and P4) might have resulted from donor-acceptor pair recombinations. Fig. 5 shows the PL transition mechanism in the h-BN film. The PL peak at $360.4 \mathrm{~nm}(3.44 \mathrm{eV}$,

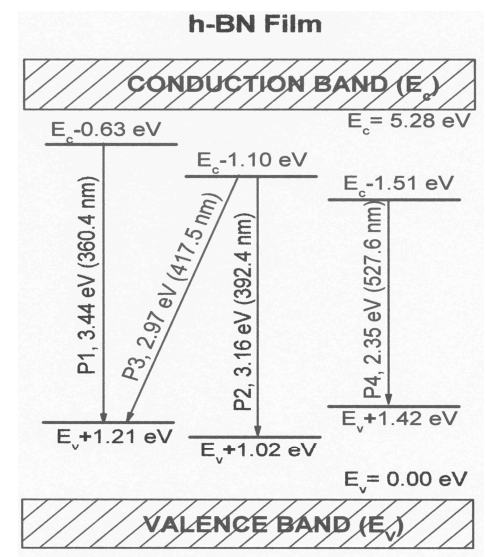

Fig. 5. Proposed energy band scheme for the radiative mechanism of the h-BN film at $10 \mathrm{~K}$. 
P1) might have been a consequence of the radiative transition from the donor level at $0.63 \mathrm{eV}$ below the conduction band to the acceptor level at $1.21 \mathrm{eV}$ above the valence band of the h-BN film. Similar explanations can be extended for the PL peaks at $392.4 \mathrm{~nm}(3.16 \mathrm{eV}, \mathrm{P} 2), 417.5$ $\mathrm{nm}(2.97 \mathrm{eV}, \mathrm{P} 3)$, and $527.6 \mathrm{~nm}(2.35 \mathrm{eV}, \mathrm{P} 4)$.

\section{Conclusion}

The h-BN films were grown on quartz plates by spaying a $\mathrm{BN}$ powder dispersed- $\mathrm{H}_{3} \mathrm{BO}_{4}-\mathrm{BCl}_{3}$-ethyl alcohol solution. Subsequently, they were dried off, and heated to $1070{ }^{\circ} \mathrm{C}$. Next, they were crystallized into a hexagonal structure with the lattice constants of $\mathrm{a}=2.5044 \pm 0.0025 \AA$ and $\mathrm{c}=$ $6.6562 \pm 0.0054 \AA$. The optical energy band gap of the $h-$ $\mathrm{BN}$ film was $5.28 \mathrm{eV}$. There were three donor levels at activation energies of $0.63,1.10$, and $1.51 \mathrm{eV}$, and three acceptor levels with activation energies of 1.02, 1.21, and $1.42 \mathrm{eV}$. The four PL peaks at $10 \mathrm{~K}$ were at $360.4 \mathrm{~nm}(3.44$ $\mathrm{eV}), 392.4 \mathrm{~nm}(3.16 \mathrm{eV}), 417.5 \mathrm{~nm}(2.97 \mathrm{eV})$, and 527.6 $\mathrm{nm}(2.35 \mathrm{eV})$. Based on analysis, these PL peaks were the results of donor-acceptor pair recombinations.

\section{Acknowledgements}

This work was supported by the Korea Research Foundation Grant (KRF-2006-013-D00226).

\section{References}

[1] A. Zunger, A. Katzir, and A. Halperin, "Optical properties of hexagonal boron nitride", Phys. Rev. B 13, p.5560, 1976.

[2] D. M. Hoffman, G. L. Doll, and P. C. Eklund, “Optical reflectance study of the electronic structure of acceptor-type graphite intercalation compounds", Phys. Rev. B 30, p.6051, 1984.
[3] O. Stenzel, J. Hahn, M. Roder, A. Ehrlich, S. Prause, and F. Richter, "Electron microscopic analysis of cubic boron nitride films deposited on fused silica", phys. stat. sol. (a) 158, p.281, 1996.

[4] C. W. Ong, K. F. Chan, and C. L. Choy, "Optical absorption and transport mechanisms of dual ion-beamdeposited boron-rich boron nitride films", Thin Solid Films 388, p.217, 2001.

[5] A. Katzir, J. T. Suss, A. Zunger, and A. Halperin, "Point defects in hexagonal boron nitride. I. EPR, thermoluminescence, and thermally-stimulated-current measurements", Phys. Rev. B 11, p.2370, 1975.

[6] V. V. Lopatin and F. V. Konusov, "Continuum optical radiation produced by $\mathrm{H}^{+}$and $\mathrm{He}^{+}$ion bombardment of pyrolytic boron nitride", J. Phys. Chem. Solids 53, p.847, 1992.

[7] H. J. Song, S. H. Yun, W. T. Kim, and C. S. Yoon, J. "Photoluminescence and TSC of $\mathrm{TlGaS}_{2}$ single crystal", Korean Phys. Soc. 26, p.617, 1993.

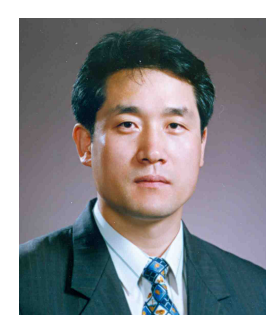

Moon-Seog Jin received his Ph. D. in Physics from Chonnam National University in 1994. His research interest is semiconductor materials. Currently, he is a professor in the Dept. of Optometry and Optic Science in Dongshin University

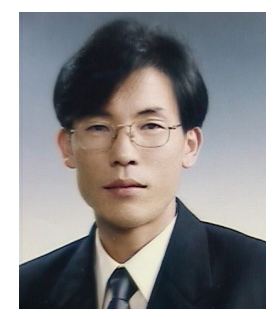

Nam-Oh Kim received his B.S and M.S degree in Electrical Engineering from Chosun University in 1996, and 1998, respectively. His research interests are in semiconductor and electric materials. Currently, he is a professor at the Dept. of Electricity at Chosun University College of Science \& Technology. 